\section{The Effect of Methadone on Sexual Function in Men Dependent on Opiates}

\section{Nasim Fooladi ${ }^{2}$, Roohollah Jirdehi ${ }^{1 *}$, Zahra Mohtasham-Ami- ri $^{3}$ and Fatemeh Pakzad ${ }^{1}$}

${ }^{1}$ Guilan University, Rasht, Iran

${ }^{2}$ Mazandaran University of Medical Sciences, Sari, Iran

${ }^{3}$ Guilan University of Medical Sciences, Rasht, Iran

\begin{abstract}
Sexual dysfunction disorder is one of the most common problems faced by opiates dependent people. Accordingly, the present study examined the effect of methadone maintenance treatment on sexual function in men dependent on opiates before and after 6 months of treatment. In this study, a cross-sectional method was used in which 60 married men addicted to drug referring to rehabilitation centers in Rasht were selected and evaluated. Initially, the patient's demographic data were collected and then, their sexual function before and after treatment was assessed by the international index of erectile function. The results showed that erectile function, sexual satisfaction and overall sexual satisfaction increased significantly after 6 months of treatment, while the orgasmic function of people addicted to opiates decreased significantly during this period. Overall, results showed an average prevalence of sexual dysfunction disorder at the beginning of treatment and improvement of some sexual function components after treatment with methadone.
\end{abstract}

Keywords: Methadone Maintenance Treatment (MMT); Opiates; Sexual dysfunction disorder

\section{Introduction}

Addiction is one of the most miserable problems of modern societies. Drug abuse disorders and their unpleasant consequences account for one of the major public health problems throughout the world [1]. Drug dependence is a chronic recurrent disorder whose onset and persistence are affected by the interaction of genetic, psychological, social and environmental factors. Addiction not only leads to severe and profound damages to individuals' mental and physical health, but also results in social harms, such as divorce, crime and unemployment. Many studies have shown that most patients with drug abuse suffer from multiple physical illnesses

*Corresponding author: Roohollah Jirdehi, Guilan University, Rasht, Iran, Tel: +98 09112319135; E-mail: jirdahi@gmail.com

Citation: Fooladi N, Jirdehi R, Mohtasham-Amiri Z, Pakzad F (2016) The Effect of Methadone on Sexual Function in Men Dependent on Opiates. J Community Med Public Health Care 3: 013.

Received: December 10, 2015; Accepted: January 29, 2016; Published: February 12, 2016
There are different modalities for treatment of drug dependence including detoxification and maintenance treatment. In these modalities, various types of narcotic and non-narcotic drugs are used. Methadone is one of the most widely used narcotic drugs in this field [5]. Methadone is a synthetic compound as an alternative narcotic drug that is prescribed in drug dependence treatment whose aim is reduction in damage resulted from drug abuse [6]. Methadone maintenance treatment was introduced in 1964 by Vincent Dole and Marie Nyswander making the hypothesis that high doses of methadone consumption leads to eliminate drug craving and to interrupt any kind of euphoria resulted from arbitrary use of drugs by fostering cross tolerance. So, opiates consumers get rid of preoccupation with drug and drug seeking behavior. This in turn, causes they can conduct their energies to more productive ways [7]. Despite there lative safety of long-term methadone has been proved completely, due to lack of complete tolerance induced to some of its effects, the side effects associated with drug used develops. Some of these effects, such as euphoria and drowsiness decrease rapidly in the first weeks of treatment, but other effects, such as constipation, excessive sweating, decreased sexual desire and sexual dysfunction continue or several months after taking medication [8].

Sexual dysfunction disorder due to persistent and chronic use of opiates has been one of the serious problems that drug abusers are facing with [9]. According to the DSM definition, sexual dysfunction is a kind of disorder in sexual desire and psychosocial changes that Impact on the sexual response cycle, cause distress and interpersonal difficulties, and include lack of sexual desire, sexual aversion, disorder of sexual stimulation and orgasm, dyspareunia and vaginism [10]. Sexual dysfunctions in men are inability to have a satisfying sex relationship, including erectile dysfunction, premature ejaculation, delayed ejaculation and retrograde ejaculation [11]. Many studies have examined the relationship between changes in sexual function, and drug abuse, and the results suggest that different drugs have different effects on sexual function. The drugs abused affect sexual function in various ways. Many of them with low doses boost sexual function by reducing or inhibiting anxiety or mood temporary boosting, but congestion erection and orgasm and ejaculation abilities are disrupted with continued use of them [12]. Sexual dysfunction disorder resulted from using opiates can be occurred in all phases of sexual arousal (erection), plateau (phase of sexual excitement), and orgasm (ejaculation) in the sexual responses cycle [13]. In addition, study by [14] found sexual dysfunction could cause relapse to drug use in patients who are undergoing detoxification treatment. The issue taken into consideration in various studies in other countries is the different remedial effects on sexual function in the treatment of addiction to opiates. The studies by Brown et al., [15], Spring et al., [16], Bliesener et al., [17] and Hanbury et al., [18] showed that there was a difference in sexual dysfunction between after and before methadone treatment. On the other hand, evidence indicates the relationship between the treatment failure and incidence of sexual dysfunction that this factor, in turn, leads to reduced compliance in drug dependent patients [19]. Thus, given to the commonness and difference in opiate addiction treatment effect on sexual function, as 
well as, lack of research in this field, the present study was conducted in order to examine sexual dysfunction in drug addicts in receiving MMT.

\section{Methods}

The present study was cross-sectional. The statistical population included all male addicts treated with MMT in remedial centers of drug dependence and abuse in Rasht in 1393 (Persian date) (2014). The study statistical sample included 60 addict persons that referred to addiction remedial center of Tavallodino in Rasht that were under MMT. Study inclusion criteria included being male and married, history of drug dependency, undergoing MMT, absence of organic disease affecting sexual function and not taking any medication affecting sexual function. The subjects were evaluated during a 6-month period (the first and second measurement was conducted at the beginning of treatment and 6 months afterwards, respectively).

Demographic questionnaire and International Index of Erectile Function (IIEF) questionnaire were used for collecting data which had 15 questions designed by Rosen, Alth of and Giuliano, and measures five main areas of sexual function including orgasmic function, sexual desire, erectile function, intercourse satisfaction and overall satisfaction of individuals. The maximum score of erectile dysfunction is 30 (while the highest score in the evaluation of orgasmic dysfunction was 10 , sexual satisfaction 15 and overall sexual satisfaction 10). The test was based on the Likert rating scale.

\section{Results}

The study included 60 married men who were under MMT. The subjects were between 26 and 47 years old with mean of 32.46 years. Pre-treatment drug using duration was 5-19 years with mean of 11.46 years.

T-test was used for inference about each subscale of sexual function at the beginning of treatment and after treatment. The results are shown in table 1 . As it can be seen in the table 1, the mean of the subjects erectile function at the beginning of the treatment was18.50, representing the average prevalence of this problem in the subjects at that time. At the end of 6 months of treatment, erectile function increased to 23.33. The results analysis indicated that erectile function following MMT improved significantly $(\mathrm{p}<0.01)$.

Mean orgasmic function of the subjects at the beginning of treatment and after six months of methadone treatment was 7.86 and 7.10, respectively, indicating a decline of orgasmic function after
MMT. The results showed that the orgasmic function after MMT decreased significantly $(\mathrm{p}<0.05)$.

At the beginning of treatment and after 6 month of treatment, mean score on sexual desire was 6.35 and 6.30, respectively, indicating that there is no significant difference among sexual desire of people in the two times $(\mathrm{p}>0.05)$.

The subject's sexual satisfaction means score at the beginning of treatment and after 6 months of MMT was 9.08 and 9.88, respectively. The study's results showed that sexual satisfaction increased significantly after treatment $(\mathrm{p}<0.05)$.

At the beginning of treatment and after 6 months of MMT, overall sexual satisfaction scores mean of the subjects was 7.26 and 8.28, respectively. The analysis results showed that overall sexual satisfaction significantly increased after treatment $(\mathrm{p}<0.01)$.

\section{Discussion}

The present study aimed at evaluating sexual dysfunction in men using drug sat the beginning of treatment and 6 months after wards. The study results showed that the severity of some aspects related to sexual dysfunction reduced among patients using drugs after MMT. This can be related to gradual effects of methadone on the sexual hormones system during treatment which is supporting that the pharmacokinetic properties of methadone lead to adaptation and normalization of the endocrine system and body neuroendocrine function [20].

The results also showed that erection problems during the use of MMT were moderate that reached mild level after receiving MMT. This result was consistent with Hallinan et al., [8] and Babakhanian et al., [9] investigating that the erectile dysfunction in opiates addict men under MMT and showed that these patients had a serious impairment in erectile function and treatment with methadone was effective significantly in reducing their erectile dysfunction. Although the improvement in erectile function among participants in the study can be obtained from direct effect of methadone, however, further studies are required to develop a precise causal relationship. The mentioned results contrasted with Roshanpajhu et al., [12] that studied the effect of MMT on sexual function in men dependent on opiates and showed there was no significant difference in erectile function of patients taking medication between before and after treatment.

Also, the study showed that there was significant difference in subjects orgasmic function between before and after treatment, so that orgasmic function before treatment was higher than after it.

\begin{tabular}{|c|c|c|c|c|c|c|c|}
\hline Subscales Sig & Samples & & & Mean Deviation & Standard Deviation & $\mathbf{T}$ & Df \\
\hline \multirow{2}{*}{ Erectile function } & Before treatment & 60 & 18.50 & 3.27 & \multirow{2}{*}{-7.62} & \multirow{2}{*}{59} & \multirow{2}{*}{0} \\
\hline & After treatment & 60 & 23.33 & 4.16 & & & \\
\hline \multirow{2}{*}{ Orgasmic function } & Before treatment & 60 & 7.86 & 1.75 & \multirow{2}{*}{3.28} & \multirow{2}{*}{59} & \multirow{2}{*}{0.002} \\
\hline & After treatment & 60 & 7.10 & 1.73 & & & \\
\hline \multirow{2}{*}{ Sexual desire } & Before treatment & 60 & 6.35 & 1.68 & \multirow{2}{*}{0.30} & \multirow{2}{*}{59} & \multirow{2}{*}{0.76} \\
\hline & After treatment & 60 & 6.30 & 0.96 & & & \\
\hline \multirow{2}{*}{ Sexual satisfaction } & Before treatment & 60 & 9.08 & 2.10 & \multirow{2}{*}{-2.37} & \multirow{2}{*}{59} & \multirow{2}{*}{0.02} \\
\hline & After treatment & 60 & 9.88 & 1.73 & & & \\
\hline \multirow{2}{*}{ Overall satisfaction } & Before treatment & 60 & 7.26 & 1.32 & \multirow{2}{*}{-6.72} & \multirow{2}{*}{59} & \multirow{2}{*}{0} \\
\hline & After treatment & 60 & 8.28 & 1.23 & & & \\
\hline
\end{tabular}


Citation: Fooladi N, Jirdehi R, Mohtasham-Amiri Z, Pakzad F (2016) The Effect of Methadone on Sexual Function in Men Dependent on Opiates. J Community Med Public Health Care 3: 013.

Palha et al., [21] investigating sexual function in 101 patients dependent on heroin also showed that $60 \%$ of the patients had problems in reaching orgasm. After methadone treatment, orgasmic function in these people came down, indicating a decrease in methadone effectiveness or increase in opiates on increasing orgasmic performance of people.

In this study, there was no difference in sexual desire between before and after treatment, reflecting the fact that methadone taking does not make a major change in sexual desire, although it somewhat decreased. Accordingly, Teusch et al., [22] examined the patterns of sexual dysfunction in schizophrenic, neurotic patients, and patients receiving MMT and showed that patients taking methadone had decreased sexual desire, with this reason that it may be caused by methadone and reduced serum testosterone levels.

On the other hand, the present study showed there was significant difference in sexual satisfaction and overall satisfaction of patients receiving MMT before and after initiating it, and it seems that the use of methadone led to remarkable increase both in sexual satisfaction and patient's overall satisfaction. In addition to methadone effect, psychological interventions, educating mental skills and lifestyle changes during treatment can improve interpersonal relationships and quality of life. This leads to a significant increase in sexual and overall satisfaction.

\section{Conclusion}

Overall, the results indicate that erectile function, sexual satisfaction and overall satisfaction of the subjects increased significantly after treatment with methadone and orgasmic function decreased significantly. Also, the results showed that no significant difference observed among the subjects sexual desire. However, having sexual disorders might be associated with many reasons other than addiction, therefore it is hard to conclude that Methadone intake would result in sexual disorders in addicts. Further research has to be done on this issue by controlling variables such as drug usage history, previous sexual experience, income, education and living standards.

\section{Limitations}

The study limitations are as follows: low volume of the sample, being single sex of the sample, ignoring age as a factor affecting sexual function and only paying attention to the patient saying that should be considered in generalizing the results.

\section{Acknowledgement}

We appreciate all the participants in this study, therapists and colleagues of dependency and drug abuse treatment center of Tavallodino.

\section{References}

1. Daley DC, Marlatt GA (2005) Relapse prevention. In: Lowinson JH, Ruiz P, Millman RB, Langrod JG (eds.). Substance Abuse: A Comprehensive Textbook. (4thedn), Lippincott Williams \& Wilkins, Philadelphia, USA. Pg no: 772 785 .

2. Mokri A (2013) A guide to treat methamphetamine use based on the revised version of Matrix model. (1stedn), Ghazal, Tehran, Iran.

3. Khademi A, AleYasin A, Agha-Hosseini M, Dadras N, Asghari Roodsari A, et al. (2006) Psychometric properties of Sexual Function questionnaire: evaluation of an Iranian sample (Persian). Iranian Journal of Reproductive Medicine 4: $23-28$.
4. Khademi A, Alleyassin A, Amini M, Ghaemi M (2008) Evaluation of sexual dysfunction prevalence in infertile couples. J Sex Med 5: 1402-1410.

5. Purnaghash Tehrani S (2009) The comparison of effectiveness of MMT and MD on anxiety and depression signals in drug abuse persons. Journal of Psychological Studies 4: 81-100.

6. Kheirabadi, Reza G, Salehi, Mehrdad, Golafshan, et al. (2012) Sexual Dysfunction of Men on Methadone Maintenance Treatment MMT and Its, Relation with Methadone Dose and Blood Level of Testosterone and Prolactine. Medical Journal of Tabriz University of Medical Sciences \& Healt 34: 68-73.

7. Kaplan HI, Saddock BJ, Grebb JA (1999) Comprehensive textbook of psychiatry. (7thedn), Lippincott Williams \& Wilkins, Philadelphia, USA. Pg: 10551057.

8. Hallinan R, Byrne A, Agho K, McMahon C, Tynan P, et al. ( 2008 ) Erectile dysfunction in men receiving methadone and buprenorphine maintenance treatment. J Sex Med 5: 684-692.

9. Babakhanian M, Eqlima, Rahbar G (2011) A preliminary study on sexual functioning disorders among men with opiates abuse, methadone maintenance therapy. Journal of Social Sciences 5: 86-98.

10. Basson R, Berman J, Burnett A, Derogatis L, Ferguson D, et al. (2000) Report of the international consensus development conference on female sexual dysfunction: definitions and classifications. J Urol 163: 888-893.

11. Nikoobakht M (2003) Sexual disorders in men and women. Tehran University of Medical Sciences, Tehran, Iran.

12. Roshanpajhu M, Khodaei MR, Tafi E, Rezaei O, Nazeri-Astaneh A, et al. (2012) The Effect of Methadone on Sexual Function in Male Dependent to Opiates: Special Issue Addiction Researches 12: 65-71.

13. Quaglio G, Lugoboni F, Pattaro C, Melara B, G.I.C.S, et al. (2008) Erectile dysfunction in male heroin users, receiving methadone and buprenorphine maintenance treatment. Drug Alcohol Depend 94: 12-18.

14. Patel K, Hellstrom WJ (2009) Central regulation of ejaculation and the therapeutic role of serotonergic agents in premature ejaculation. Curr Opin Investig Drugs 10: 681-690.

15. Brown R, Balousek S, Mundt M, Fleming M (2005) Methadone maintenance and male sexual dysfunction. J Addict Dis 24: 91-106.

16. Spring WD Jr, Willenbring ML, Maddux TL (1992) Sexual dysfunction and psychological distress in methadone maintenance. Int J Addict 27: 13251334.

17. Bliesener N, Albrecht S, Schwager A, Weckbecker K, Lichtermann D, et al. (2005) Plasma testosterone and sexual function in men receiving buprenorphine maintenance for opioid dependence. J Clin Endocrinol Metab 90: 203206.

18. Hanbury R, Cohen M, Stimmel B (1977) Adequacy of sexual performance in men maintained on methadone. Am J Drug Alcohol Abuse 4: 13-20.

19. Brown RT, Zueldorff M (2007) Opioid Substitution with Methadone and Buprenorphine: Sexual dysfunction as a side effect of therapy. Heroin Addict Relat Clin Probl 9: 35-44.

20. Martin J, Payte JT, Zweben JE (1991) Methadone maintenance treatment: a primer for physicians. J Psychoactive Drugs 23: 165-176.

21. Palha AP, Esteves M (2002) A study of the sexuality of opiate addicts. J Sex Marital Ther 28: 427-437.

22. Teusch L, Scherbaum N, Böhme H, Bender S, Eschmann-Mehl G, et al. (1995). Different patterns of sexual dysfunctions associated with psychiatric disorders and psychopharmacological treatment. Results of an investigation by semistructured interview of schizophrenic and neurotic patients and methadone-substituted opiate addicts. Parmacopsychiatry 28: 84-92. 